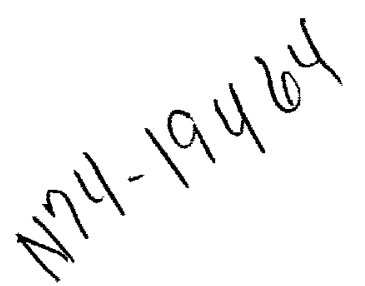

\title{
28. Optical Properties of Atmospheric Dust From Twilight Observations
}

\author{
N. B. Divari, Y. I. Zaginayilo, \\ AND L. V. KovalchuK \\ Odessa Polytechnic Institute \\ Odessa, U.S.S.R.
}

\begin{abstract}
Three methods of approximation are described and are used to separate the primary twilight brightness from the observed brightness. Photoelectric observations obtained at the Majaky Observatory in 1968-69 (Zaginayilo) are combined with observations from a balloon and from Pic-du-Midi Observatory (Link et al., 1967) to derive the atmospheric scattering phase functions at $0.87 \mu$ and $0.58 \mu$ as a function of height.

Comparison of these data with data for a Rayleigh atmosphere provide information on the optical properties of dust in the upper atmosphere.
\end{abstract}

$\mathrm{T}_{\mathrm{s}, \mathrm{s}}$ HE OBSERVED BRIGHTNESS $B_{\text {obs }}$ of the twilight I sky may be considered as the sum of the primary brightness $B_{1}$ and the secondary one $B_{2}$ :

$$
B_{\text {obs }}=B_{1}+B_{2}
$$

For investigation of the optical properties of the Earth's atmosphere it is necessary to separate the primary brightness $B_{1}$ from the observed brightness $B_{\text {obs. }}$. It is a difficult problem. As there is no widely adopted method now for separating the primary brightness, we have determined this brightness by using three approximation mothods.

The first method was proposed by V. G. Fessenkov (1968a) and utilizes obscrvations in two points of the solar meridian with zenith distances $z=70^{\circ}$. By this method the brightness of the secondary twilight in the observation point is determined from observations in the other point (the base point) lying in the Earth's shadow and having the zenith distanee $z=70^{\circ}$.

This method may be extended to points of the sky with zenith distances different from $z=70^{\circ}$. Values of coefficient $k$ are listed in table 1. The coefficient $k$ is used for computations of the secondary brightness $B_{2}(z)$ in the observation point from the sccondary brightness $B_{2}{ }^{\prime}\left(70^{\circ}\right)$ in the base point $z=70^{\circ}, A=180^{\circ}$ by the formula $B_{2}(z)=k B_{2}^{\prime}\left(70^{\circ}\right)$.

In the second method, proposed by Divari (1971), we use another coefficient $k_{1}$ which is defined by the ratio $B_{1}\left(B_{1}+B_{2}\right)=k_{1}$. This coefficient $k_{1}$ may also be found from the computations for the standard atmosphere. Valucs of this coefficient are listed in table 2 .

These values found according to the data of Divari and Plotnikova (1965), for corresponding wavelengths and atmospheric transmission coefficients may be considered only as a first approximation.

The third method for the determination of the primary brightness is V. G. Fessenkov's method of observations of the point of the sky with zenith distance $z=80^{\circ}$ and azimuth $A=30^{\circ}$ (from the Sun azimuth). This method requires measurement of intensity, degree and angle of polarization of the light of the twilight sky in the quoted point (Fessenkov, 1968b). 
TABle 1.-Values of Coefficient $\mathrm{k}=\mathrm{B}_{2}(\mathrm{z}) / \mathrm{B}_{2}{ }^{\prime}\left(\gamma 0^{\circ}\right)$ used for $\mathrm{A}=0^{\circ}$ at Wavelengths $\lambda=0.37 \mu$ and $\lambda=0.58 \mu$ With Corresponding Almospheric Transmission Coefficients $\mathrm{p}=0.59$ and $\mathrm{p}=0.89$ (Base Point $\mathrm{z}=70^{\circ}, \mathrm{A}=180^{\circ}$ )

\begin{tabular}{|c|c|c|c|c|c|c|c|c|}
\hline & \multicolumn{4}{|c|}{$\lambda=0.37 \mu, p=0.59$} & \multicolumn{4}{|c|}{$\lambda=0.58 \mu, p=0.89$} \\
\hline & $4^{\circ}$ & $6^{\circ}$ & $10^{\circ}$ & $14^{\circ}$ & $4^{\circ}$ & $6^{\circ}$ & $10^{\circ}$ & $14^{\circ}$ \\
\hline $80^{\circ}$ & 1.31 & 1.46 & 1.51 & 1.54 & 2.12 & 2.55 & 2.63 & 2.73 \\
\hline 70 & 0.891 & 1.47 & 1.37 & 1.37 & 1.10 & 2.00 & 2.11 & 2.17 \\
\hline 60 & 0.809 & 1.30 & 1.25 & 1.25 & 0.673 & 1.19 & 1.24 & 1.26 \\
\hline 40 & 0.583 & 0.897 & 0.846 & 0.842 & 0.337 & 0.574 & 0.579 & 0.580 \\
\hline 20 & 0.451 & 0.650 & 0.592 & 0.585 & 0.222 & 0.368 & 0.366 & 0.363 \\
\hline 0 & 0.385 & 0.535 & 0.503 & 0.496 & 0.185 & 0.304 & 0.303 & 0.300 \\
\hline-20 & 0.388 & 0.535 & 0.557 & 0.555 & 0.195 & 0.324 & 0.328 & 0.323 \\
\hline-40 & 0.458 & 0.650 & 0.746 & 0.749 & 0.254 & 0.427 & 0.438 & 0.436 \\
\hline-60 & 0.600 & 0.873 & 0.988 & 0.998 & 0.403 & 0.684 & 0.711 & 0.710 \\
\hline
\end{tabular}

TABLE 2.-Values of Coefficient $\mathrm{K}_{1}=\mathrm{B}_{1} /\left(\mathrm{B}_{1}+\mathrm{B}_{2}\right)$ Use for the Wavelengths $\lambda=0.37$ and $\lambda=0.58 \mu$ (Transmission Coefficients $\mathrm{p}=0.59$ and $\mathrm{p}=0.89$ ) and Computed According to the Data of Divari and Plotnikova (1965) for $\mathrm{A}=0^{\circ}$

\begin{tabular}{|c|c|c|c|c|c|c|c|c|}
\hline \multirow{2}{*}{$z$} & \multicolumn{4}{|c|}{$\lambda=0.37 \mu, p=0.59$} & \multicolumn{4}{|c|}{$\lambda=0.58 \mu, p=0.89$} \\
\hline & $4^{\circ}$ & $6^{\circ}$ & $10^{\circ}$ & $14^{\circ}$ & $4^{\circ}$ & $6^{\circ}$ & $10^{\circ}$ & $14^{\circ}$ \\
\hline $80^{\circ}$ & 0.70 & 0.79 & 0.66 & 0.43 & 0.80 & 0.76 & 0.52 & 0.49 \\
\hline 70 & 0.75 & 0.74 & 0.24 & 0.16 & 0.75 & 0.62 & 0.22 & 0.22 \\
\hline 60 & 0.73 & 0.68 & 0.10 & $8.010^{-2}$ & 0.72 & 0.55 & 0.12 & 0.12 \\
\hline 40 & 0.69 & 0.59 & $3.4 \quad 10^{-2}$ & $3.410^{-2}$ & 0.69 & 0.46 & $5.610^{-2}$ & $5.610^{-2}$ \\
\hline 20 & 0.65 & 0.52 & $2.310^{-2}$ & $2.210^{-2}$ & 0.66 & 0.40 & $3.710^{-2}$ & $3.510^{-2}$ \\
\hline 0 & 0.63 & 0.47 & $1.610^{-2}$ & $1.510^{-2}$ & 0.65 & 0.36 & $2.710^{-2}$ & $2.310^{-2}$ \\
\hline-20 & 0.63 & 0.44 & $9.410^{-3}$ & $8.010^{-3}$ & 0.64 & 0.33 & $1.610^{-2}$ & $1.210^{-2}$ \\
\hline-40 & 0.62 & 0.35 & $4.110^{-3}$ & $2.710^{-3}$ & 0.63 & 0.27 & $7.610^{-3}$ & $4.310^{-3}$ \\
\hline-60 & 0.51 & 0.13 & $7.910^{-4}$ & $2.710^{-4}$ & 0.56 & 0.14 & $1.410^{-3}$ & $3.010^{-4}$ \\
\hline
\end{tabular}

Having the primary brightness $B_{1}$ as the function of the Sun depression $g\left(g=z-90^{\circ}\right.$, where $z$ is the zenith distance of the Sun) or of the effective hright of the twilight ray $h$, it is possible to determine the scattering coefficient $\sigma(\theta, h)$ of the atmosphere as a function of the scattering angle $\theta$ and the height $h$ above the surface of the Earth. This procedure is described by Divari (1971).

Knowing $\sigma(\theta, h)$, we can find the volume scattering coefficient $\Sigma(h)$ (or cxtinction coeff- cient) according to

$$
\Sigma(h)=\int \sigma(\theta, h) d \omega
$$

where $d \omega$ is an clement of solid angle and integration is over all the angles.

For the purpose of comparing the three foregoing methods of separating primary brightness $B_{1}$ and determining the scattering cocfficients, we have used the photoclectric obscrvations of twilight sky made by Y. I. Zaginayilo in 1968- 
1969 at the observatory Majaky near Odessa (USSR).

Figure 1 shows the observed brightness $B_{\text {obs }}$ in the solar meridian at the points $z=70^{\circ}$ and $z=-60^{\circ}$ (the minus sign indicates that the observed point is on the antisolar side of the Sun meridian) and the primary brightnesses $B_{1}^{(1)}$ and $B_{1}{ }^{(2)}$ found by the two methods above. As may be seen, the $B_{1}{ }^{(1)}$ and $B_{1}{ }^{(2)}$ at the point $z=70^{\circ}$ are nearly equal in the interval $2^{\circ}$ to $8^{\circ}$ of the Sun deprossion. For $g>8^{\circ}$, the second method leads to lower values than the first one $\left(B_{1}{ }^{(2)}<\right.$ $\left.B_{1}^{(1)}\right)$. At the point $z=-60^{\circ}$ the deviation of $B_{1}^{(2)}$ from $B_{1}^{(1)}$ begins earlier, approximately at $g=4^{\circ}$.

Figure 2 shows the primary brightnesses $B_{1}{ }^{(1)}$ and $B_{1}{ }^{(2)}$ at the wavelength $\lambda=0.58 \mu$ obtained from the observed brightness $B_{\mathrm{obs}}$ at the point $z=30^{\circ}$ of the Sun meridian. In the

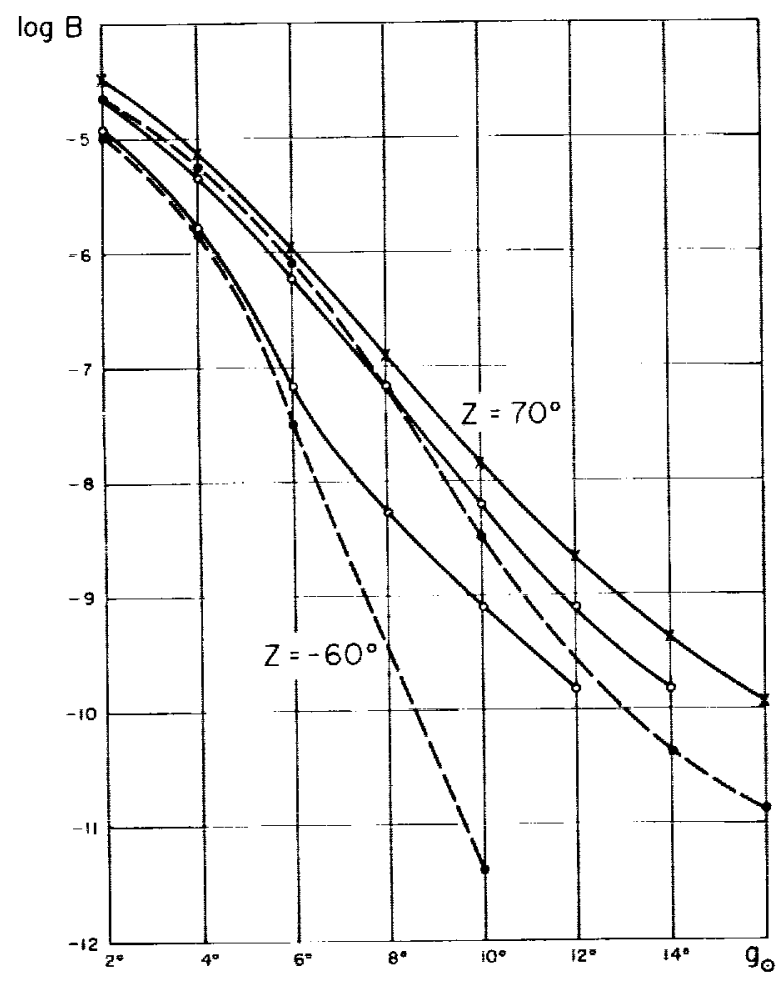

Figure 1.- Iog of the observed brightness $B_{\mathrm{oba}}$ at the points $Z=70^{\circ}$ (crosses) and $Z=-60^{\circ}$ of the solar meridian and primary brightnesses $B_{1}{ }^{(1)}$ (open circles) and $B_{1}{ }^{(2)}$ (black dots) for the same point of the sky, plotted against $g_{\odot}$, the solar depression angle. same figure we have shown the brightness $B_{1}(25)$ $(\lambda=0.53 \mu)$ which was obtained by F. Link, L. Neuzil, and I. Zacharov (1967) by means of balloon observations at the height of $25 \mathrm{~km}$ above the Earth's surface. By crosses we have shown the observed brightnosses, obtained by Link et al. (1967) from the observation (at the wavelength $\lambda=0.53 \mu$ ) at the Pic-du-Midi observatory $(H=2.9 \mathrm{~km})$. It seems from comparing the data presented in figure 2 that $B_{1}{ }^{(2)}$ gives a more realistic approximation of primary brightnesses than $B_{1}{ }^{(1)}$.

In figure 3 we have shown the phase functions $\sigma(\theta)$ for $\lambda 0.37 \mu$ and $\lambda 0.58 \mu$ obtained from the $\sigma(\theta, h)$ by averaging in the height interval 30 to $130 \mathrm{~km}$. The vertical bars indieate the standard errors. The same figure shows the Rayleigh scattering function $\sim 1+\cos ^{2} \theta$. As seen from figure 3 , the intensity of scattered light increases

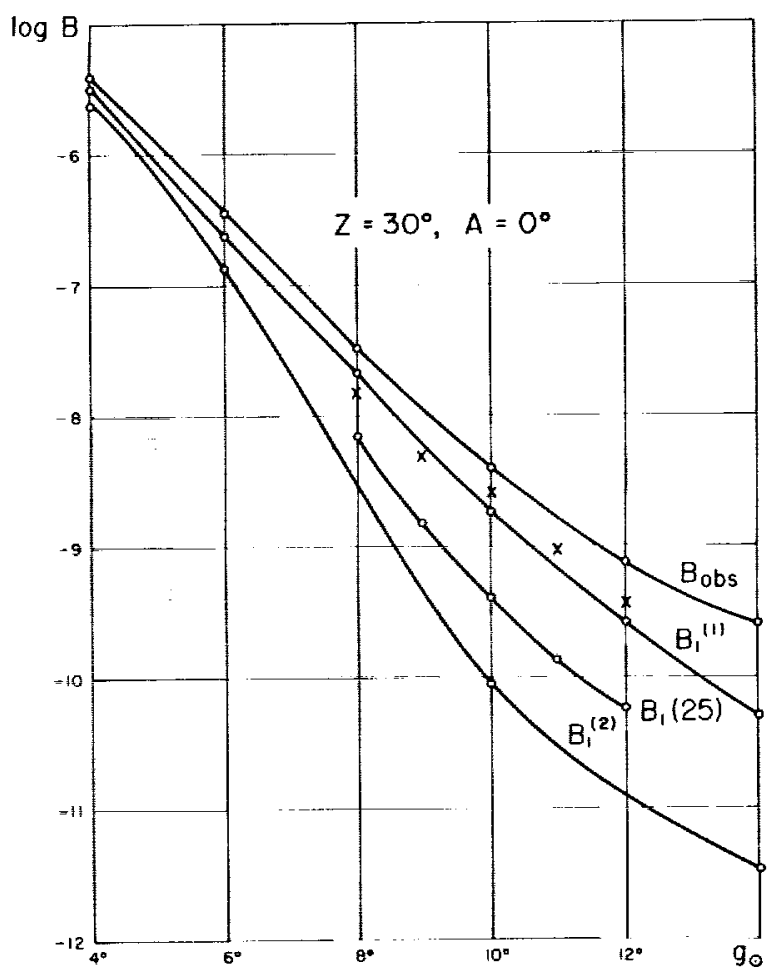

Figure 2.- Iog of the observed brightness $B_{\text {obs }}$ and primary brightnesses $B_{1}^{(1)}$ and $B_{1}{ }^{(2)}$ at wavelengths $\lambda$ $0.58 \mu$ from observations at the point $Z=30^{\circ}$ of the sun meridian. $B_{1}(25)$ is primary brightness according to Link et al. (1967) from balloon observation. Crosses are their observations of brightness at the Pic-du-Midi. Abscissae are solar depression angles $g_{\odot}$. 
with the decrease of angle $\theta$ steeper than for Rayleigh scattering. It seems that it is the manifestation of the influcnce of acrosols on the optical properties of atmosphere. It may be pointed out that the increase of scattering intensity with the decrease of the scattering angle $\theta$ is flatter at $\lambda 0.37 \mu$ than at $\lambda 0.58 \mu$.

Figure 3 also shows the acrosol phase function $\sigma_{a}(\theta)$ obtained from the atmospheric scattering coefficient $\sigma(\theta)$ by subtracting the Rayleigh scattering coefficient $\sigma_{R}(\theta)$, calculated for standard atmosphere CIRA, 1965. In the same figure we show the aerosol phase function obtained by E. V. Pyaskorskaya-Fessenkova (1970) from observations of the day skylight. As may be scen, there is a good agreement between the phase functions obtained from twilight and daytime observations.

The scattering coefficient $\sigma(\theta)$ was defined from observations in the sun meridian for the interval of seattering angles $\theta=25^{\circ}$ to $155^{\circ}$. Since for the determination of the extinction coefficient $\Sigma$ it is necessary to integrate $\sigma(\theta)$ over the interval $\theta$ from $\theta=0^{\circ}$ to $\theta=180^{\circ}$, we extrapolate this function from $\theta=25^{\circ}$ to $\theta=0^{\circ}$ and from

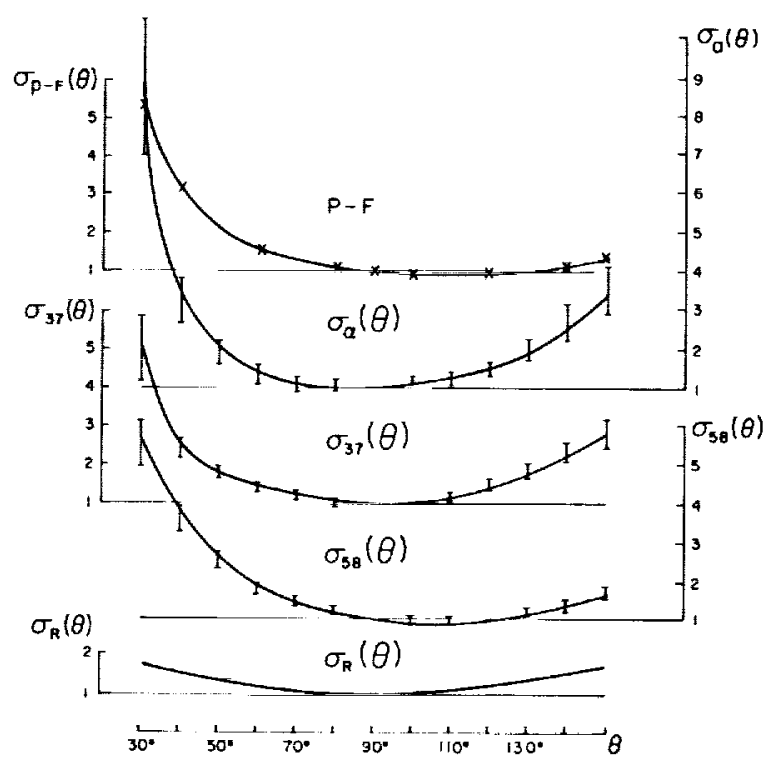

FIGCRE 3.-Atmospheric phase function $\sigma_{37}(\theta)$ for $\bar{\lambda} 0.37 \mu$ and $\sigma_{i s}(\theta)$ for $\lambda 0.58 \mu$ and aerosol phase function $\sigma_{a}(\theta)$ for $\lambda 0.37 \mu . P-F$ is acrosol phase function according to Pyaskovskaya-Fessenkova (1970) for $\lambda$ $0.546 \mu . \sigma_{R}(\theta)$ is Raylcigh phase function for $\lambda 0.37 \mu$. $\theta=155^{\circ}$ to $\theta=180^{\circ}$. The extrapolation from $\theta=25^{\circ}$ to $\theta=0^{\circ}$ was made by taking into account the results found by G. Newkirk and J. A. Eddy (1963) for the small angles from the stratospheric coronagraph observations. The extrapolation in the angle interval $160^{\circ}$ to $180^{\circ}$ is made with sufficient certainty and the error of this extrapolation according to our estimations can reach a few percent of the value of $\Sigma$.

Figure 4 shows the extinction coefficients $\Sigma^{(1)}, \Sigma^{(2)}$ and $\Sigma^{(3)}$ obtained for the primary

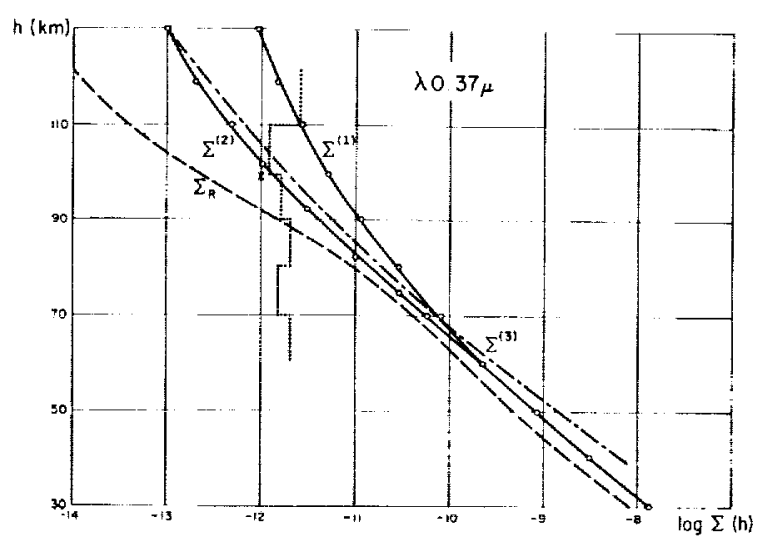

Figure 4.-Extinction coefficient $\Sigma^{(1)}, \Sigma^{(2)}$ and $\Sigma^{(3)}$ obtained from the primary brightnesses $B_{1}^{(1)}, B_{1}^{(2)}$ and $B_{1}{ }^{(3)}$ at the wavelength $\lambda 0.37 \mu$. Point $X$ is the value according to Mikirov (1963). Dotted line portrays extinction coefficient according to Fiocco and Columbo (1964).

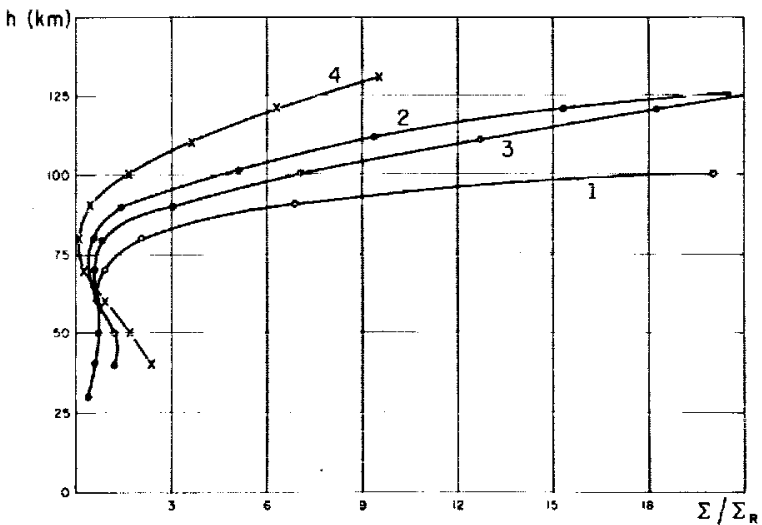

Figure 5.-Ratios $\Sigma / \Sigma_{R}$. Curve 1 is ratio obtained from $B_{1}{ }^{(1)}$ in the Sun meridian. Curve 2 is ratio obtained from $B_{1}{ }^{(2)}$ in the Sun meridian. Curve 3 is ratio obtained from $B_{1}{ }^{(2)}$ at the point $Z=80^{\circ}, A=30^{\circ}$. Curve 4 is ratio obtaincd from $B_{1}{ }^{(3)}$ at the point $Z=80^{\circ}$, $A=30^{\circ}$. 
brightnesses $B^{(1)}, B^{(2)}$ and $B^{(3)}$ at wavelength $\lambda 0.37 \mu$, computed by the three forcgoing methods. In the same figure there is the Rayleigh extinction coefficient $\Sigma_{R}$ computed for atmosphere model CIRA 1965. The dotted line indicates the extinction coefficient found for the heights 60 to $120 \mathrm{~km}$ by G. Fiocco and G. Colombo (1964) by means of laser observations. The cross indicates the extinction coefficient found by $\Lambda$. E. Mikirov (1963) by means of rocket measurements.

To present the importance of the aerosols in the atmospheric scattering, we have computed the ratio $\Sigma / \Sigma_{R}$ for different heights from 30 to
$125 \mathrm{~km}$. As is seen from figure 5 , the values of this ratio are near unity in the height interval 30 to $80 \mathrm{~km}$ and rapidly increase above $80 \mathrm{~km}$. It secms that this phenomenon is the result of the light scattered by dust which penetrates into the Earth's atmosphere from the interplanetary space or arises from meteor ablation.

The presented data and the results of comparing the twilight data with data obtained by other methods permit the conclusion that the twilight method offers good possibilities for investigation of the optical properties of the upper atmosphere and can be used for monitoring the dust concentration in the upper atmosphere.

\section{REFERENCES}

Drvari, N, B., 1971. The results of investigation of dust in the upper atmosphere by the twilight method, Space Research XI, 351-355.

Divari, N. B., ANd Plotnikova, L. I., 1965. Computed brightness of the twilight sky, Astron. Zhurn. Akad. Nauk., SSSR, 42, 1090-1103.

Fessenkov, V. G., 1968a. Atmosfernaya optika, Nauka Press, Moscow, 96. Translated and published by Plenum Publ. Corp, New York, "Atmospheric optics," edited by N. B. Divari, 1970,90 .

- 1968 b. Almosfernaya optika, Nauka Press, Moscow, 175.

Frocco, G., And Colombo, G., 1964. Optical radar results and meteoric fragmentation, $J$. Geophys. Res., 69, 1795-1805.

Lnk, F., Neusil, L., AND Zacharov, I, 1967. Mesures simultances du ciel crepusculaire in ballon et au sol, Ann. Geophys., 23, 207-216.

Mikinov, A. E., 1963. Aerosol scattering coefficient measurements at 80-100 km, Space Research $I I I, 155-161$.

NFW KIRK, G., JR., AND EDDY, J. A., 1963. Influx of meteor particles in the upper atmosphere of the Earth as determined from stratospheric coronagraph observations, Space Research III, 143-154.

Pyaskovskaya-Fessenkova, F. V., 1970. Atmosfernaya optika, Nauka Press, Moscow, 94 (to be translated by Plenum Publ, Corp.). 
